\title{
Database Dry Meadows and Pastures of Switzerland
}

\author{
Christophe Hunziker
}

\begin{abstract}
Dry meadows and pastures are habitats which are very rich in species. As they are also highly endangered, the Federal Council has recorded them in an inventory, as required under article 18a of the Federal Act on the Protection of Nature and Cultural Heritage (NCHA; SR 451). The Database Dry Meadows and Pastures of Switzerland (GIVD ID EU-CH-006) contains 13,724 relevés (28 sq. meters) from whole Switzerland, ranging from 200 to 2,800 m. Relevés were assessed between 1995 and 2009 from 45 different people.
\end{abstract}

Keywords: dry grassland; Switzerland; vegetation.

GIVD Database ID: EU-CH-006

Last update: $2012-05-06$

\section{Database Dry Meadows and Pastures of Switzerland}

Scope: The dry meadows and pastures vegetation database contains almost 14,000 relevés from whole Switzerland, ranging from 200 to 2,800 m. Relevés were assessed between 1995 and 2009 from 45 different people.

Status: completed and continuing

Period: $1995-2009$

Database manager(s): Christophe Hunziker (c.hunziker@humagis.ch)

Owner: Federal Office for the Environment FOEN

Web address: http://www.bafu.admin.ch/schutzgebiete-inventare/07849/index.html?lang=de

Availability: according to a specific agreement Online upload: no Online search: no

Database format(s): MS Access Export format(s): MS Access, Excel

Publication: Eggenberg, S., Dalang, T., Dipner, M., Mayer, C., 2001, Cartography and evaluation of dry grasslands sites of national importance, Technical report, Schriftenreihe Umwelt SRU-325-E, Federal Office for the Environment (FOEN), Bern, 252 P.

Plot type(s): normal plots

Plot-size range: $28-28 \mathrm{~m}^{2}$

Non-overlapping plots: 13,724

Estimate of existing plots: 13,724

Completeness: $100 \%$

Total plot observations: 13,724

Number of sources: 1

Valid taxa: 1,665

Countries: $\mathrm{CH}: 100.0 \%$

Forest: $0 \%$ - Non-forest: aquatic: 0\%; semi-aquatic: 0\%; arctic-alpine: $12 \%$; natural: 29\%; semi-natural: 59\%; anthropogenic: <1\%

Guilds: all vascular plants: $100 \%$

Environmental data: altitude: $100 \%$; other soil attributes: $100 \%$

Performance measure(s): cover: $100 \%$

Geographic localisation: GPS coordinates (precision $25 \mathrm{~m}$ or less): $69 \%$; point coordinates less precise than GPS, up to $1 \mathrm{~km}$ : $31 \%$

Sampling periods: $1990-1999: 36.0 \% ; 2000-2009: 64.0 \%$

Information as of 2012-07-12; further details and future updates available from http://www.givd.info/ID/EU-CH-006

Christophe Hunziker (c.hunziker@ humagis.ch)

Maillefer \& Hunziker, Rue des Pêcheurs 8a, 1400 Yverdon-les-Bains, SWITZERLAND 